\title{
MANEJO DAS ÚlCERAS DO PÉ DIABÉTICO NO CONTEXTO DA ATENÇÃO PRIMÁRIA À SAÚDE (APS): UMA REVISÃO INTEGRATIVA
}

\author{
MANAGEMENT OF DIABETIC FOOT ULCERS IN THE CONTEXT OF PRIMARY \\ HEALTH CARE (PHC): AN INTEGRATIVE REVIEW \\ Renata de Cássia Coelho Pires ${ }^{1}$ \\ Adriana Dias Lucena ${ }^{2}$ \\ Jhennyfer Barbosa de Oliveira Mantesso ${ }^{3}$ \\ Claudilene Sousa Fortaleza ${ }^{4}$
}

RESUMO: O Diabetes Mellitus é considerado um agravo crônico bastante recorrente nas comunidades, cujas úlceras em membros inferiores se caracterizam como uma complicação comum, de alto custo e tratamento prolongado. Os profissionais da Estratégia Saúde da Família, frequentemente se encontram em manejo desta complicação em seus territórios de abrangência, através de cuidados específicos, que incluem desde aspectos preventivos até o tratamento tópico e ações educativas para o autocuidado. Desta forma, com objetivo de identificar as principais formas de manejo das úlceras do pé diabético no contexto da Atenção Primária à Saúde (APS), através dos profissionais da Estratégia Saúde da Família, realizou-se uma revisão integrativa da literatura, com dados coletados através de artigos científicos por busca na base de dados Scielo e (Scientific Electronic Library Online) e BVS. Os resultados demonstraram que a principal forma de manejo diz respeito aos aspectos preventivos, através de ações educativas e orientações quanto ao autocuidado, cujo sucesso está relacionado à recorrência de tais práticas. No que diz respeito ao diagnóstico precoce e modos de rastreio, houve déficit nas ações de avaliação clínica por parte das equipes de saúde da família e ainda, pouco comprometimento quanto ao autocuidado pelos pacientes, cujas ações comprometidas dos profissionais da APS são essenciais para mudança deste cenário.

Palavras-chave: Diabetes Mellitus. Manejo clínico. Atenção Primária à Saúde.

\footnotetext{
' Graduada em Enfermagem e Obstetrícia pela Universidade Estadual do Maranhão (UEMA), Mestre em Doenças Tropicais pela Universidade Federal do Pará (UFPA) e doutoranda em Saúde Pública pela Universidade de Ciências Empresariais e Sociais (UCES), Buenos Aires, Argentina. Docente da Universidade Federal do Maranhão (UFMA), curso de Enfermagem. E-mail: renatacoelhopires@hotmail.com

${ }^{2}$ Graduada em Enfermagem pelo Instituto Tocantinense Presidente Antônio Carlos (ITPAC), Mestre em Doenças Tropicais pela Universidade Federal do Pará (UFPA) e doutoranda em Saúde Pública pela Universidade de Ciências Empresariais e Sociais (UCES), Buenos Aires, Argentina. Docente da Universidade Federal do Maranhão (UFMA), curso de Enfermagem.

${ }^{3}$ Graduada em Enfermagem pela Universidade Federal do Maranhão (UFMA), Especialista em Saúde da Mulher pelo Instituto Nordeste de Educação Superior e Pós-graduação (INESPO).

${ }^{4}$ Graduada em Serviço Social pela Universidade do Sul do Maranhão (UNISULMA), Especialista em processos educacionais na saúde com ênfase em metodologia, pelo Hospital Osvaldo Alemão (PROADI), mestranda em Saúde da mulher e da criança pelo Instituto Fernandes Figueira (IFF).
} 
ABSTRACT: Diabetes Mellitus is considered a chronic disease that is very recurrent in communities, whose ulcers in the lower limbs are characterized as a common complication, of high cost and prolonged treatment. Family Health Strategy professionals are often managing this complication in their territories, through specific care, which includes everything from preventive aspects to topical treatment and educational actions for self-care. Thus, in order to identify the main forms of management of diabetic foot ulcers in the context of Primary Health Care (PHC), through the professionals of the Family Health Strategy, an integrative literature review was carried out, with data collected through scientific articles by searching the database Scielo e (Scientific Electronic Library Online) and BVS. The results showed that the main form of management concerns preventive aspects, through educational actions and guidance on self-care, whose success is related to the recurrence of such practices. With regard to early diagnosis and screening methods, there was a deficit in the clinical evaluation actions by the family health teams and, still, little commitment regarding the patients' selfcare, whose committed actions of the PHC professionals are essential to change this scenery.

Keywords: Diabetes Mellitus. Clinical management. Primary Health Care.

\section{INTRODUÇÃO}

O Diabetes Mellitus (DM) é uma doença crônica que persiste com grande relevância ao setor saúde em razão de seu forte aspecto biopsicossocial. É capaz de gerar sérios impactos de morbimortalidade na população brasileira, ocasionando diversos tipos de complicações se não tratada adequadamente. Dentre elas, umas das mais comuns é a úlcera do pé diabético, cujo manejo clínico apropriado é essencial para prevenir desfechos incapacitantes, tal como as amputações.

Trata-se de um distúrbio hormonal caracterizado por hiperglicemia persistente decorrente da deficiência na produção e/ou na ação da insulina (OLIVEIRA; MONTENEGRO JÚNIOR; VENCIO, 2017). Atualmente é considerada uma doença de alta prevalência, onde a Organização Mundial da Saúde (OMS) calcula que 422 milhões de adultos vivam com DM e que I,6 milhões de mortes são decorrentes dessa patologia anualmente no mundo (SILVEIRA et al., 2018)

A Atenção Primária à Saúde (APS) é uma das responsáveis por acompanhar pessoas com DM, visando reduzir complicações, incapacidades e internações decorrentes da doença (NEVES et al., 2018). No contexto domiciliar, esse acompanhamento é frequentemente 
realizado por equipe multiprofissional, em especial médicos e enfermeiros da Estratégia Saúde da Família (ESF), que atuam em territórios delimitados, com população adscrita e cadastrada.

Os profissionais atuantes na ESF devem considerar a importância do manejo adequado deste agravo, em virtude do aumento de prevalência do DM e, por conseguinte, de sua complicação microvascular mais frequente, a Neuropatia Diabética (ND), destacando-se a importância de conhecer suas principais manifestações clínicas, os métodos de investigação disponíveis e o tratamento proposto, a fim de proporcionar um diagnóstico precoce com possibilidade de prevenção de progressão da doença e suas complicações (NASCIMENTO; PUPE; CAVALCANTI, 2016).

Deste modo, a presente pesquisa objetiva investigar as principais formas de manejo das úlceras do pé diabético no contexto da APS, através da descrição das principais condutas adotadas pelos profissionais das equipes de ESF quanto à prevenção, orientações quanto ao autocuidado e manejo tópico. Para isso, o termo pé diabético será designado para indicar as diversas alterações e complicações ocorridas, isoladamente ou em conjunto, nos pés e nos membros inferiores dos pacientes diabéticos, cujo resultado dessa complicação é um elevado custo humano e financeiro. O pé diabético em estágio terminal, necrosado e infectado é uma condição frequente em todos os serviços de emergência, resultado de ações precárias de prevenção e de meses ou anos de assistência inespecífica (CAIFA et al., 2oII), o que reforça sobre a importância da devida atuação da APS.

As úlceras do pé diabético representam complicações passíveis de prevenção, desde que haja um comprometimento do paciente quanto às condutas de autocuidado e disponibilidade de uma rede assistencial em saúde resolutiva. Tal assistência deve ocorrer através de medidas de cuidados e conforto para os pés, atenção integral ao portador da diabetes com controle do índice glicêmico e ampliação da rede de atenção à saúde. Logo, acredita-se que o fortalecimento da prática de cuidado com os pés e a busca ativa dos portadores de diabetes na atenção primária à saúde pode ser uma importante estratégia para melhoria do cenário nacional (FERNANDES et al., 2020).

Deste modo, para o alcance do objetivo proposto, realizou-se uma revisão integrativa da literatura, em artigos científicos e manuais acerca do assunto. A base de dados utilizada foi 
Scielo (Scientific Electronic Library Online) e BVS com as seguintes abordagens de busca: Diabetes Mellitus; Pé diabético; Atenção Primária à Saúde; Manejo clínico e suas combinações na língua portuguesa: "Pé diabético na Atenção Primária"; "Manejo clínico do pé diabético e Pé diabético na Atenção Primária à Saúde”.

\section{ASSISTÊNCIA EM SAÚDE NAS ÚLCERAS DO PÉ DIABÉTICO NO CONTEXTO DA ATENÇÃO PRIMÁRIA À SAÚdE (APS)}

O DM se trata de uma doença de ampla ocorrência na população brasileira, cujo acompanhamento se caracteriza como uma rotina aos profissionais atuantes na Estratégia Saúde da Família (ESF). É considerado um dos principais problemas de saúde pública no Brasil e no mundo e sua prevalência vem aumentando em decorrência do aumento da expectativa de vida, sendo que nos países em desenvolvimento sua ocorrência não se limita somente às idades mais avançadas. Assim, evidencia-se a necessidade da implantação de políticas públicas de saúde que permitam melhorar a qualidade de vida dos indivíduos acometidos e seus familiares, uma vez que esta patologia é considerada Condição Sensível à Atenção Primária, onde a abordagem adequada neste nível de atenção à saúde permite evitar hospitalizações e mortes decorrentes de suas complicações (BRASIL, 2013).

Em decorrência dessa alta prevalência e por se tratar de um agravo crônico, o DM requer um acompanhamento adequado por parte dos serviços de saúde, visto que, suas complicações são graves e incluem um alto risco de morbimortalidade. Assim, passa a configura-se como um cenário alarmante e sugere a necessidade de investir em melhorias na qualidade do manejo dessas morbidades, principalmente na Atenção Primária à Saúde (APS), por ser o nível de atenção responsável pela detecção precoce das condições crônicas e acompanhamento daqueles já diagnosticados, evitando suas complicações e a necessidade de internação (SILVA et al., 2019).

Levando em consideração as principais atribuições da APS, também chamada de Atenção Básica ( $\mathrm{AB}$ ), pode-se afirmar que a mesma se estrutura por meio de um conjunto de ações de saúde individuais, familiares e coletivas que promovem prevenção a doenças e proteção à saúde, com práticas de cuidado integrado e focadas em territórios específicos. Para 
isso, equipes multiprofissionais de saúde atuam com responsabilidade sanitária pelo seu território adscrito (BRASIL, 2017). Médicos, enfermeiros, agentes comunitários de saúde e de endemias e técnicos de enfermagem, integram estas equipes, assim como, são os responsáveis pela assistência direta aos portadores de DM, sendo o manejo clínico conduzido por médicos e enfermeiros, particularmente.

Deste modo, uma das principais atribuições de médicos e enfermeiros da ESF consiste na avaliação regular dos pés das pessoas portadoras de DM. No entanto, os demais profissionais das equipes, mesmo de nível técnico, devem estar habilitados para o reconhecimento precoce de alterações do pé diabético. No âmbito da APS, este cuidado deve ser sistematizado, de modo que toda população nesta condição seja devidamente acompanhada. É fundamental organizar o acesso das pessoas com DM para que a avaliação dos pés seja regular e abrangente para o indivíduo e, ao mesmo tempo, eficiente para a equipe, em termos do tempo e dos recursos despendidos. Após o adequado levantamento da necessidade de avaliações na população de referência, é útil a equipe manter uma planilha atualizada com a data e o resultado do último exame dos indivíduos com DM na comunidade. Essa planilha permite à equipe monitorar a data prevista de retorno das pessoas, facilitando a busca ativa quando ela se fizer necessária (DUNCAN; GOLDRAICH, CHUERI, 2013).

Outro aspecto a ser considerado diz respeito à importância do incentivo pelo profissional da APS quanto às práticas de autocuidado, no qual se destaca a atuação da enfermagem, visto que, no atendimento das pessoas com DM, que apresentam algum tipo de complicação em membros inferiores, a equipe de enfermagem observa a necessidade de melhorar a qualidade das orientações para o autocuidado, auxiliando-as na melhoria da qualidade de vida e na minimização de danos à saúde (PADILHA et al., 2017).

Um estudo conduzido sobre autocuidado com os pés em portadores de DM, a respeito do autoexame nos pés, inspeção de calçados antes da utilização e secagem dos espaços interdigitais depois de lavá-los, demonstrou escores muito próximos ao desejável, desde que, haja um estímulo adequado para o desenvolvimento dessas habilidades por parte dos profissionais de saúde (GOMIDES et al., 2013). 
Assim, o principal desafio da prevenção de complicações decorrentes do pé diabético reside em fortalecer as ações para efetivar o autocuidado mediante o ensino de habilidades e atitudes instrutivas, empregando dinâmicas diferenciadas que possibilitem promover a aprendizagem com vistas a reduzir a incidência dessa complicação (MOREIRA et al., 2020). Portanto, os profissionais de saúde da ESF constituem fator imprescindível nas atividades de educação em saúde para o enfrentamento do pé diabético e seu pior desfecho: as amputações.

Como fatores desencadeantes do pé diabético, tem-se a neuropatia periférica como a principal causa de complicação tardia nos pés dos pacientes diabéticos. As deformidades decorrentes da paralisia muscular intrínseca, da perda da sensibilidade protetora dos pés e da destruição osteoarticular no pé e tornozelo são as três ocorrências mais comuns que predispõem ao aparecimento das úlceras de pressão nos pés (FERREIRA et al., 20Io).

As úlceras em membros inferiores em portadores de DM costumam ser bastante ameaçadoras à qualidade de vida de seus portadores, especialmente quando associadas a fatores que dificultam o processo de cicatrização. Quando a úlcera do pé é complicada por uma infecção, a combinação pode ser um risco para o membro e até para a vida. A infecção é definida clinicamente, mas as culturas ajudam na identificação dos agentes patogênicos responsáveis e assim a instituição de terapia antimicrobiana. No entanto, embora a antibioticoterapia possa curar a infecção isoladamente, não cicatriza a úlcera, requerendo uma atuação para a correção de outros fatores associados (focos de pressão anômala, isquemia do membro) para alcance da cicatrização (DUARTE e GONÇALVES, 20II).

A depender da gravidade do quadro em paciente com úlceras diabéticas, as medidas terapêuticas podem ocorrer em âmbito hospitalar ou ambulatorial, nesse contexto, a APS também pode acolher indivíduos nestas condições, que estejam estáveis, porém com necessidade de acompanhamento específico mediante o surgimento do pé diabético. Desta forma, uma das atribuições iniciais da equipe de saúde se dá em classificar a gravidade da infecção da úlcera a fim de determinar o tratamento adequado para o paciente, que pode ser medicamentoso (antibioticoterapia) e/ou cirúrgico (encaminhamento). O tratamento imediato e adequado da infecção pode diminuir seu efeito destrutivo e controlar o foco séptico (NEVES et al., 2013). 
Desta forma o tratamento das úlceras diabéticas em membros inferiores está relacionado primariamente com o grau de neuropatia, presença de infecções, isquemia e distúrbios mecânicos ou estruturais, cabendo enfatizar que os serviços de atenção básica devem dispor de atuação vigilante quanto à prevenção e detecção precoce deste agravo, além do manejo tópico adequado, visto que estes pacientes muitas vezes se encontram em acompanhamento domiciliar. Medidas simples de realização de curativos tradicionais com uso apenas de soro fisiológico, desbridantes e gaze estéril, precedidos da degermação periúlcera são efetivos no tratamento da úlcera diabética, desde que acompanhados do necessário alívio da pressão. Coberturas especiais devem ser avaliadas em situações especificas e sempre com a análise do custo/beneficio (CAIFA et al., 2oir).

Assim, a presente pesquisa apresenta uma abordagem sobre as principais formas de assistência empregadas aos portadores de DM e/ou pé diabético no contexto da APS, através de levantamento bibliográfico, sobre condutas realizadas por médicos e enfermeiros pertencentes à ESF, além de discorrer sobre os principais benefícios frente ao processo de cicatrização e/ou prevenção de amputações que devem ser realizadas no âmbito domiciliar.

\section{MÉTODOS}

Trata-se de uma pesquisa de revisão integrativa da literatura cuja finalidade é subsidiar um fundamento teórico acerca do manejo do pé diabético no contexto da APS, através da atuação dos profissionais das equipes de ESF em território brasileiro. Para isto, seguiram-se as seguintes etapas, conforme o Quadro I, a partir da seguinte questão norteadora: Quais as principais condutas desempenhadas pelos profissionais de saúde da ESF acerca do manejo do pé diabético em seus aspectos preventivos, de diagnóstico e tratamento? Sendo considerados profissionais de saúde todos os integrantes das equipes de saúde da família (enfermeiro, médico, técnico de enfermagem e agente comunitário de saúde).

Assim, para a seleção da literatura científica pertinente, foi realizada uma busca na base de dados selecionada, referente aos últimos io anos (2010 a 2020) com os seguintes descritores: pé diabético; atenção primária à saúde; diabetes mellitus e as seguintes combinações: "manejo clínico do pé diabético"; "pé diabético na atenção primária à saúde"; 
"tratamento pé diabético". Foram incluídos no estudo os artigos escritos em português, entre os anos de 2010 a 2020, disponíveis na íntegra e online na base de dados SciELO e Biblioteca Virtual em Saúde - BVS, sendo incluídos apenas artigos que abordassem os seguintes aspectos: descrição de profissionais envolvidos na assistência (médicos e enfermeiros); local de práticas tendo como cenário a APS; descrição de benefícios ou dificuldades encontradas na assistência prestada aos portadores de DM e que identificassem as atividades de assistência prestadas aos pacientes com DM e/ou pé diabético.

Quadro I - Descrição das etapas da revisão integrativa da literatura.

\begin{tabular}{|c|c|}
\hline $\begin{array}{l}\text { I" etapa: Formulação do problema/questão norteadora. } \\
\text { "Quais as principais condutas desempenhadas pelos prof } \\
\text { ao portador do pé diabético em seus aspectos preventivos, }\end{array}$ & $\begin{array}{l}\text { issionais de saúde da ESF acerca da assistência } \\
\text { de diagnóstico e tratamento?" }\end{array}$ \\
\hline 2- etapa: Artigos encontrados & Scielo \\
\hline & 45 artigos \\
\hline $3^{\text {a }}$ etapa: Artigos selecionados & 23 \\
\hline $\begin{array}{l}4^{\mathbf{a}} \text { e } 5^{\mathbf{a}} \text { etapa: Artigos utilizados para apresentação dos } \\
\text { resultados. }\end{array}$ & $2 \mathrm{I}$ \\
\hline
\end{tabular}

Fonte: Dados de pesquisa, 2020/2I.

A coleta de dados ocorreu nos meses de dezembro de 2020 a Fevereiro de 2021, com auxílio de roteiro para coleta das seguintes informações: identificação do artigo; objetivos; desenho metodológico; resultados; limitações e conclusões. A análise do material selecionado buscou responder a questão norteadora e alcançar os objetivos propostos na presente pesquisa, com manutenção das ideias centrais de cada estudo realizado.

\section{RESULTADOS E DISCUSSÕES}

A quantidade final de publicações selecionadas para a fundamentação das discussões e alcance do objetivo da pesquisa foram 21 (artigos) distribuídos nos seguintes anos: 2012 (or); 2014 (04); 2017 (05); 2019 (04); 2020 (07). Quanto aos objetivos, os estudos se centraram nos seguintes aspectos acerca da assistência prestada aos portadores de DM e/ou Pé diabético: Custos financeiros sobre o tratamento do pé diabético; avaliação da atenção ao DM na perspectiva da APS; conhecimentos e práticas acerca de medidas preventivas do pé diabético; grau de conhecimento dos pacientes com DM acompanhados pela ESF; fatores associados ao desenvolvimento de pé diabético; o papel das intervenções educativas para prevenção do pé 
diabético na comunidade; propostas de cuidados ao portador de pé diabético em razão da pandemia de covid-ı;; alterações sensoriais típicas em portadores de DM; ações de enfermagem no cuidado ao pé diabético; a importância da visita domiciliar em pacientes que sofreram amputações; síntese de evidências sobre o controle e prevenção do pé diabético; a neuropatia periférica e sua influência ao pé diabético e grau de risco para ulcerações dos pés em portadores de DM.

A análise dos artigos permitiu verificar que as principais formas de assistência oferecida aos pacientes diabéticos acompanhados pela APS, estão relacionadas ao seu aspecto preventivo, com a realização de atividades educativas para o autocuidado. Não houve estudos que apontassem a assistência quanto ao manejo tópico das lesões de membros inferiores de pacientes com DM. No entanto, apesar dos estudos enfatizarem acerca das atividades educativas como principal forma de cuidado, a avaliação periódica dos pés pelos profissionais de saúde também se constitui medida fundamental, conforme sugerido no Protocolo Clínico e Diretrizes Terapêuticas do DM tipo I (2020), como estratégia de diagnóstico precoce. Neste, há a recomendação que o exame dos pés de indivíduos com esta enfermidade, deve ocorrer logo após o diagnóstico e seguir de forma anual. Essa avaliação consiste na observação de possíveis alterações anatômicas do pé diabético, hidratação, coloração, temperatura e distribuição de pêlos, integridade de unhas e pele e possíveis alterações neurológicas incluindo sensibilidade, reflexos tendíneos e função motora, visto que, os mecanismos pelos quais as lesões no pé do paciente diabético ocorrem podem ser neuropáticos, vascular (isquêmico) ou misto (BRASIL, 2020). Portanto, o acompanhamento pontual e sistemático é essencial para uma assistência de qualidade.

No que diz respeito ao cumprimento deste parâmetro, estudo de Silva et al. (2019) acerca da qualidade da assistência ofertada à idosos com DM acompanhados pela ESF em Belo Horizonte (MG), apresentou como pior parâmetro a avaliação do pé diabético, com apenas 2,2\% dos pacientes sendo avaliados periodicamente. Já no estudo de Tavares et al. (2014), realizado em uma Unidade Básica de Saúde em Pernambuco, a qualidade da assistência foi caracterizada como regular, pois todas as 40 equipes realizavam atividades educativas e 22 realizavam o exame dos pés dos pacientes cadastrados. Considerando ainda, aspectos 
relacionados ao exame dos pés, no estudo de Fernandes et al. (2020), que tratou da prevalência e os fatores associados às ações de prevenção das úlceras dos pés em pacientes diabéticos no Brasil, houve uma baixa proporção de realização do exame dos pés em portadores de diabetes, com importantes desigualdades entre as regiões brasileiras (maior na região Sudeste e menor na região Centro-Oeste), associando ainda a carência da realização do exame e da recomendação para realizá-lo, às piores condições sociais e econômicas.

Assim, tais resultados demonstram uma assimetria na oferta de ações de saúde voltadas a prevenção e cuidado ao portador de pé diabético, pois o exame clínico dos pés é atribuição dos profissionais de saúde, que precisam estar cientes de sua importância. Neste contexto, estudo de Vargas et al. (2017) envolvendo enfermeiros da ESF em uma cidade do Sul do Brasil, evidenciou que o conhecimento destes profissionais sobre os cuidados com a pessoa com DM é parcial, superficial e fragmentado, não possibilitando ações adequadas ao cuidado, especialmente, na detecção dos riscos para o desenvolvimento do pé diabético e para realizar a avaliação do exame dos pés. Sendo ainda, suas condutas consideradas ineficazes, porque os enfermeiros não realizam, de forma sistematizada, os cuidados básicos para a prevenção de complicações. Deste modo, há um alerta sobre possíveis falhas e fragilidades nas práticas assistenciais vinculadas especialmente à APS, que não se mostram adequadas às necessidades de saúde desta população.

Arruda et al. (2019), também realizou um estudo envolvendo Enfermeiros da ESF de Teresina, PI, cujos resultados são semelhantes quanto às práticas assistenciais deficientes. Onde, nenhum enfermeiro apresentou conhecimento satisfatório para a prevenção do pé diabético e, acerca da autoavaliação do conhecimento, 48,9\% dos enfermeiros o consideravam regular. Verificou-se, ao analisar os itens sobre a prevenção do pé diabético, melhor desempenho para o monofilamento e pé neuropático, com menor desempenho para exame físico. Quanto à classificação do conhecimento, os profissionais apresentaram conhecimento insatisfatório $(45,6 \%)$ e conflitante $(54,4 \%)$. Logo, há a necessidade premente da implementação de ações que envolvam educação permanente em saúde, assim como atividades de atualização profissional e uso de protocolos assistenciais que possam garantir um acompanhamento mais eficaz aos diabéticos, visto que se trata de um agravo crônico. 
Inseridas ao cenário das atividades que devem ser empregadas pelos profissionais da Estratégia Saúde da Família, além da avaliação periódica dos pés da população portadora de DM, há também a recomendação de se instituir ações de ensino para o autocuidado, como medida de promoção da saúde. Segundo Brasil (2013), em seu manual sobre Estratégias para o cuidado da pessoa com DM, a abordagem educativa para prevenção da ocorrência de ulceração nos pés e/ou sua identificação precoce, deve ser estabelecidas de acordo com os fatores de risco envolvidos, segundo categorias, que vai desde a categoria o (pacientes com baixo risco de desenvolver úlceras) até a categoria 3 (no qual as úlceras já estão presentes). Além disso, os profissionais devem avaliar o potencial para o autocuidado que são: nível de conhecimento do paciente sobre o diabetes; cuidados com os pés e as unhas (complicações agudas e crônicas de fácil identificação); comportamento do paciente com relação aos seus pés; cuidado executado pela pessoa; apoio familiar no cuidado com os pés e condições dos calçados e das palmilhas. Nestas situações, as intervenções educativas devem ser correspondentes segundo o risco ou a presença de lesões. Logo, as orientações e condutas referentes ao ensino do autocuidado, devem ser de acordo com o perfil da população acompanhada, segundo suas características e necessidades particulares, mas que atenda aos critérios acima mencionados.

Acerca do impacto que as intervenções educativas são capazes de causar, dois estudos realizados em localidades distintas (Fortaleza/CE e Sul de MG), porém com finalidades semelhantes, sobre os efeitos gerados na capacidade para o autocuidado em pacientes com DM, a partir de 02 grupos (um com oferta da intervenção educativa e o outro não) cujas orientações abrangiam alimentação saudável, atividade física, avaliação de glicemia e cuidado com os pés, apontaram os seguintes resultados: no estudo de Marques et al. (2019) a realização das intervenções educativas em uma abordagem coletiva evidenciou melhoria nos aspectos da alimentação saudável e do cuidado com os pés, antes não promovidas, conforme verificado no grupo-controle. E, em Moreira et al. (2020) a intervenção educativa de autocuidado com os pés trabalhada no grupo operativo apresentou potencial para melhorar o autocuidado com os pés das pessoas com Diabetes Mellitus tipo 2 e contribuiu para a redução dos riscos de complicações nos pés destas pessoas. É inquestionável a contribuição das atividades de educação em saúde para redução ou não ocorrência de complicações, no entanto, tais práticas, 
consideradas como indispensáveis na assistência ao paciente com diabetes, dependem que as mesmas sejam utilizadas de modo rotineiro pelas equipes de saúde, para o alcance de mudanças positivas em hábitos de vida e comportamentos de saúde.

No entanto, importante se faz ponderar, que os benefícios oriundos das intervenções educativas, também estão pautados em sua frequência, pois mudanças no estilo de vida ou a adoção de novos hábitos são adquiridas em longo prazo e requerem um estímulo sustentado e que possam repercutir em aspectos, que muitas vezes, só podem ser mensurados a médio e longo prazo, como redução de peso, melhora de índices glicêmicos, mudança de padrão alimentar, não sedentarismo entre outros.

No estudo conduzido por Arruda et al. (2020), que objetivou analisar a efetividade do autocuidado apoiado nos comportamentos em saúde em homens com DM em uma cidade do Noroeste do Paraná, realizados por enfermeiros, revelou efeitos estatisticamente significativos para prática alimentar saudável, além de resultados positivos, porém pouco significativos, quanto circunferência do quadril e percentual de gordura corporal. O que confirma que alguns tipos de benefícios em saúde, só podem ser mensurados em médio e/ou longo prazo, o que reforça o caráter imprescindível da continuidade das ações em saúde para hábitos de autocuidado que sejam realmente efetivos e positivos para a qualidade de vida.

No que diz respeito à prevenção das úlceras do pé diabético, ao se realizar uma associação entre o efeito das intervenções educativas realizadas por profissionais de saúde e a melhora da capacidade de autocuidado para prevenção do pé diabético, pôde-se compreender que há uma relação diretamente proporcional entre ambas, ou seja, quanto mais recorrentes são as ações educativas, maior é a adesão às práticas de autocuidado, assim como o nível de conhecimento acerca da doença e suas formas de prevenção e controle. Deste modo, estudos que apontem para um déficit de conhecimento em populações de indivíduos com esta enfermidade, refletem em uma assistência de saúde precária para esta condição, com ações ineficientes e em desacordo com as condições de saúde das comunidades.

De forma a corroborar tal afirmação, estudo realizado em Maringá (PR) que buscou avaliar o conhecimento da população com DM acompanhados em Unidades Básicas de Saúde sobre os modos de prevenção do pé diabético, demonstrou uma falta de conhecimento de 
medidas preventivas, mesmo nos pacientes com algum nível de instrução, em relação às possíveis complicações nos pés de pacientes diabéticos (CARLESSO; GONÇALVES; MORESCHI JÚNIOR, 2017). Logo, tais resultados se ancoram não apenas na qualidade da assistência ofertada, mas na consistência e recorrência do acompanhamento em saúde.

Outro estudo realizado em Picos (PI) com diabéticos sobre conhecimento, atitudes e práticas voltadas à prevenção do pé diabético, apontou que 49,4\% dos entrevistados não sabiam sobre as formas de higiene e critérios de observação nos pés, no entanto, em relação às atitudes, 80\% tinham disposição para executar o autocuidado (POLICARPO et al., 2014). No estudo de Menezes et al. (2017) que tratou das práticas de autocuidado em portadores de pé diabético, houve resultado semelhante, com um grau expressivo de déficit de conhecimento e autocuidado sobre calçados adequados, formato correto do corte das unhas e importância do exame dos pés. Os entrevistados referiram pouca ou nenhuma orientação sobre estes cuidados. Assim sendo, o diagnóstico precoce só é possível mediante o rastreio adequado na população portadora deste agravo, assim como a autocapacidade dos indivíduos em detectar possíveis complicações, que inclui a busca precoce por atendimento e uma APS resolutiva.

Portanto, importante se faz que todos os pacientes com DM sejam avaliados periodicamente quantos aos riscos de desenvolvimento de pé diabético. Estudos neste aspecto demonstraram os seguintes resultados: A maior parte dos pacientes acompanhados (64,1\%) pela ESF possuía alto grau de risco para o pé diabético (FORMIGA et al., 2020); houve um déficit na avaliação de sensibilidade protetora do pé diabético com uso de monofilamento, não sendo realizado como método de rastreio pela APS (BRITO et al., 2020) e usuários com DM acompanhados pela ESF apresentaram altas taxas de sinais e sintomas neuropáticos que predispõem ao surgimento de úlceras em membros inferiores (NASCIMENTO et al., 2019). Deste modo, demonstra-se a necessidade de um plano assistencial compatível com os fatores de risco identificados aos pacientes diabéticos e o emprego de medidas sustentadas para o rastreio e diagnóstico precoce.

Assim, com objetivo de manter a qualidade de vida e evitar complicações severas relacionadas ao pé diabéticas, especialmente as amputações, que comprometem a independência e comprometem o bem-estar, muitas estratégias assistenciais podem ser 
utilizadas no contexto da APS, dentre elas as visitas domiciliares, que abrange inúmeros objetivos, desde prestar assistência ao paciente no domicílio, identificar fatores de risco (individuais e familiares) e verificar a rede de apoio familiar existente. A assistência domiciliar a um paciente com pé diabético implica atenção integral a todos os fatores que influenciam na recuperação da complicação em questão (DIAS; SANTOS; OLIVEIRA, 20I7).

Evidências científicas para prevenção e controle do pé diabético na APS, sugerem um conjunto de ações, tais como capacitar profissionais da APS no rastreamento de risco do pé diabético, na orientação para o autocuidado, na coordenação do cuidado de pessoas com diabetes mellitus; estímulo ao autocuidado com aferição diária da temperatura do pé; identificar e realizar o manejo clínico de condições pré-ulcerativas e deformidades nos pés, com encaminhamento a serviços de referência na rede assistencial e oferta de pacotes de intervenções mais complexas. No entanto, ainda persistem barreiras para o seu emprego, tais como não adesão dos pacientes ao autocuidado, sobrecarga e falta de motivação dos profissionais e fragilidades da rede assistencial (SILVA et al., 2019). Logo, uma APS resolutiva é imprescindível para a redução dos impactos de morbimortalidade ocasionados pelo DM.

\section{CONSIDERAÇÕES FINAIS}

A análise a respeito do manejo clínico do pé diabético no contexto da APS evidenciou que a principal abordagem ocorreu quanto aos aspectos preventivos, através de ações de educação em saúde e orientações quanto ao autocuidado, desde que adequadamente instituídas pelas equipes de saúde, para alcance de adesão e resultados satisfatórios.

Quanto às formas de diagnóstico precoce para o surgimento do pé diabético, os métodos de rastreio preconizados pelo Ministério da Saúde, através de avaliações periódicas e sistematizadas dos membros inferiores, se mostrou deficiente e pouco realizada no contexto da APS.

Outro aspecto identificado diz respeito à capacidade de autocuidado prejudicada pela carência de instruções adequadas, associados ao não planejamento de ações baseadas nos fatores de risco para o surgimento do pé diabético. 
Tal situação surge como um alerta à saúde pública, que requer de modo urgente uma ampliação no que se refere ao emprego de práticas sistematizadas voltadas à prevenção e detecção precoce do pé diabético e suas implicações, assim como o encaminhamento precoce de casos mais graves.

Desta forma, a atuação da Estratégia Saúde da Família (ESF) emerge com um papel fundamental na mudança deste cenário, através de assistência à saúde eficaz ao portador de DM, para o controle desta enfermidade e assim reduzir seus impactos na morbimortalidade da população brasileira.

\section{REFERÊNCIAS}

ARRUDA, Guilherme Oliveira de et al. Intervenção educativa em homens com diabetes mellitus: efeitos sobre comportamentos e perfil antropométrico. Acta Paul. Enferm.(Online), p. eAPE20190128-eAPE20190128, 2020. Disponível em: https://www.scielo.br/j/ape/a/GjSZSznrwRdvYPyJwPHWmXq/. Acesso em: o7 dez. 2020.

ARRUDA, Luana Savana Nascimento de Sousa et al. Conhecimento do enfermeiro acerca dos cuidados com o pé diabético. Rev. enferm. UFPE on line, p. [I-8], 2019. Disponível em: https://periodicos.ufpe.br/revistas/revistaenfermagem/article/view/242175/33728. Acesso em: 08 dez. 2020.

BRASIL. Departamento de Atenção Básica. Estratégias para o cuidado da pessoa com doença crônica: diabetes mellitus. Brasília: Ed. Ministério da Saúde, 2013. i6op. Disponível em: https://bvsms.saude.gov.br/bvs/publicacoes/estrategias_cuidado_pessoa_diabetes_mellitus_ca b36.pdf. Acesso em: o9 dez. 2020.

BRASIL. Departamento de Gestão e Incorporação de Tecnologias e Inovação em Saúde. Protocolo Clínico e Diretrizes Terapêuticas do Diabete melito tipo I. Brasília: Ed. Ministério da Saúde, 2020. 68 p. $\quad$ Disponível em: http://conitec.gov.br/images/Protocolos/Publicacoes_MS/PCDT_DiabetesMellitus_TipoI_I SBN_19-08-2020.pdf. Acesso em: 09 dez. 2020.

BRASIL. Ministério da Saúde. Portaria no. 2.436 de 2i de setembro de 2017. Brasília: Diário Oficial [da] República Federativa do Brasil, 2017. Disponível em: https://bvsms.saude.gov.br/bvs/saudelegis/gm/2017/prt2436_22_09_2017.html. Acesso em: I0 dez. 2020.

BRITO, Jéssyca Fernanda Pereira et al. Alterações sensório-motoras e fatores associados em pacientes com diabetes mellitus. Texto \& Contexto-Enfermagem, v. 29, 2020. Disponível em: https://www.scielo.br/j/tce/a/cn8bvThfGmjfFSvvPFKG7jf/?format=pdf\&lang=pt. Acesso em: II dez. 2020. 
CAIAFA, Jackson Silveira et al. Atenção integral ao portador de pé diabético. Jornal vascular brasileiro, v. Io, n. 4, p. I-32, 20II. Disponível em: https://www.scielo.br/j/jvb/a/FJDCG 7 NGR8npLL5MbTbCczr/?format=pdf\&lang=pt. Acesso em: i8 dez. 2020.

CARLESSO, Guilherme Pereira; GONÇALVES, Mariana Helena Barboza; MORESCHI JÚNIOR, Dorival. Avaliação do conhecimento de pacientes diabéticos sobre medidas preventivas do pé diabético em Maringá (PR). Jornal Vascular Brasileiro, v. I6, n. 2, p. II3-II8, 2017 . https://www.scielo.br/j/jvb/a/gCqgpVR $3 H_{q F N}$ xnr7gbDfwF/?format=pdf\&lang=pt. Acesso em: 20 dez. 2020.

DIAS, Jucielma de Jesus; SANTOS, Fábia Luanna Leite Siqueira Mendes; OLIVEIRA, Fernanda Kelly Fraga. Visita domiciliar como ferramenta de promoção da saúde do pé diabético amputado. Rev. enferm. UFPE on line, p. 5464-5470, 2017. Disponível em: https://pesquisa.bvsalud.org/portal/resource/pt/bde-33873. Acesso em: 2I dez. 2020.

DUARTE, Nádia; GONÇALVES, Ana. Pé diabético. Angiol. Cir. Vasc., Lisboa ,v. 7,n. 2,p. 65-79,jun.20Ir. Disponível em: https://scielo.pt/pdf/ang/v7n2/v7n2ao2.pdf. Acesso em: o5 jan. 2021.

DUNCAN, M. S.; GOLDRAICH, M. A.; CHUEIRI, P. S. Cuidados longitudinais e integrais a pessoas com condições crônicas. Medicina ambulatorial: condutas de atenção primária baseadas em evidências. 4a ed. Porto Alegre: Artmed, p. 892-904, 2013.

FERNANDES, Fábia Cheyenne Gomes de Morais et al. O cuidado com os pés e a prevenção da úlcera em pacientes diabéticos no Brasil. Cadernos Saúde Coletiva, v. 28, n. 2, p. 302-310, 2020. https://www.scielo.br/j/cadsc/a/ry4MJhfG3t9MpGBrjmWgDHD/?format=pdf\&lang=pt. Acesso em: o6 jan. 2021.

FERREIRA, Ricardo Cardenuto et al. Aspectos epidemiológicos das lesões no pé e tornozelo do paciente diabético. Acta Ortopédica Brasileira, v. I8, n. 3, p. 135-141, 2010. Disponível em: https://www.scielo.br/j/aob/a/6Vk6x46r5mRpCT9fRzgtWvM/?format=pdf\&lang=pt. Acesso em: 07 jan. 202I.

FORMIGA, Natália Pinheiro Fabricio et al. Estratificação de risco para pé diabético numa população de idosos acompanhados na atenção primária. Revista Baiana de Enfermagem, v. 34, 2020.

GOMIDES, Danielle dos Santos et al. Autocuidado das pessoas com diabetes mellitus que possuem complicações em membros inferiores. Acta Paulista de Enfermagem, v. 26, n. 3, p. 289-293, 2013. Disponível em: https://www.scielo.br/j/ape/a/33wvfN3pN6VzDxnG 39 CYyLf/?format=pdf\&lang=pt. Acesso em: io jan. 202I. 
MARQUES, Marilia Braga et al. Educational intervention to promote self-care in older adults with diabetes mellitus. Revista da Escola de Enfermagem da USP, v. 53, 2019. Disponível em: https://www.scielo.br/j/reeusp/a/gs7Q8rTDjhL 3 CLsKPCQHnTj/?format=pdf\&lang=pt. Acesso em: 12 jan. 2021.

MENEZES, Luciana Catunda Gomes de et al. Pesquisa ação: práticas de autocuidado das pessoas com pé diabético. Rev. enferm. UFPE on line, p. 3558-3566, 2017 Disponível em: https://periodicos.ufpe.br/revistas/revistaenfermagem/article/view/234486/27682. Acesso em: I4 jan. 2021.

MOREIRA, João Batista et al. Efeito do grupo operativo no ensino do autocuidado com os pés de diabéticos: ensaio clínico randomizado. Revista da Escola de Enfermagem da USP, v. 54, 2020 . https://www.scielo.br/j/reeusp/a/kTnqkQxNCK 7yxzCDpkzBQQM/?format=pdf\&lang=pt . Acesso em: 25 jan. 202r.

NASCIMENTO, José William Araújo do et al. Neuropatia do pé diabético em usuários de uma unidade de saúde da família. Nursing (São Paulo), p. 3165-3168, 2019. Disponível em: http://revistas.mpmcomunicacao.com.br/index.php/revistanursing/article/view/380/36r.

Acesso em: 30 jan. 202I.

NASCIMENTO, Osvaldo José Moreira do; PUPE, Camila Castelo Branco; CAVALCANTI, Eduardo Boiteux Uchôa. Neuropatia diabética. Revista Dor, v. I7, p. 46-5I, 2016.

https://www.scielo.br/j/rdor/a/dfMvHLrCg5zrC $5 J_{5} F_{j} W_{D K w F}$ ? format=pdf\&lang=pt.

Acesso em: io fev. 202I.

NEVES, José et al. O pé diabético com infecção aguda: tratamento no Serviço de Urgência em Portugal. Revista Portuguesa de Cirurgia, n. 27, p. 19-36, 2013. Disponível em: https://revista.spcir.com/index.php/spcir/article/view/339/333. Acesso em: in fev. 202I.

NEVES, Rosália Garcia et al. Estrutura das unidades básicas de saúde para atenção às pessoas com diabetes: Ciclos I e II do Programa Nacional de Melhoria do Acesso e da Qualidade. Cadernos de Saúde Pública, v. 34, p. eooo72317, 2018. Disponível em: https://www.scielosp.org/pdf/csp/2018.v34n4/e0oo72317/pt. Acesso em: I2 fev. 2021.

OLIVEIRA, J. E. P.; MONTENEGRO JUNIOR, R. M. Vencio S. organizadores. Diretrizes da Sociedade Brasileira de Diabetes, v. 2018, 2017.

PADILHA, Ana Paula et al. Manual de cuidados às pessoas com diabetes e pé diabético: Construção por scoping study. Texto \& Contexto-Enfermagem, v. 26, n. 4, 2017. Disponível em: $\quad$ https://www.scielo.br/j/tce/a/h4wh6B55cPcPPk3s4CzdhfK/?format=pdf\&lang=pt. Acesso em: 14 fev. 202I. 
POLICARPO, Natalia de Sá et al. Conhecimento, atitudes e práticas de medidas preventivas sobre pé diabético. Revista Gaúcha de Enfermagem, v. 35, n. 3, p. 36-42, 2014. Disponível em: https://www.scielo.br/j/rgenf/a/rZZMLggGCJ8mTBrMLYnVtBd/?format=pdf\&lang=pt. Acesso em: is fev. 2021.

SILVA, Fernanda Maria et al. Síntese de evidências para políticas de saúde: prevenção e controle do pé diabético na atenção primária a saúde. 2019. Disponível em: https://www.arca.fiocruz.br/bitstream/icict/41604/2/ve_Fernanda-Silva_etal.pdf. Acesso em: 18 fev. 2021.

SILVA, Líliam Barbosa et al. Estratos de risco e qualidade do cuidado à pessoa idosa na Atenção Primária à Saúde. Revista Latino-Americana de Enfermagem, v. 27, 2019. Disponível em: https://www.scielo.br/j/rlae/a/ZPh6sNvJsypdfW63gch98Dk/?format=pdf\&lang=pt. Acesso em: 19 fev. 2021.

SILVEIRA, Victória et al. Atualizações no manejo de retinopatia diabética: revisão de literatura. Acta Méd Ligas Acad.(Porto Alegre), v. 39, n. I, p. 293-306, 2018. Disponível em: https://ebooks.pucrs.br/edipucrs/acessolivre/periodicos/acta-medica/assets/edicoes/2or8I/arquivos/pdf/24.pdf. Acesso em: 20 fev. 2021.

TAVARES, Venâncio de Sant'Ana et al. Avaliação da atenção ao diabetes mellitus em Unidades de Saúde da Família de Petrolina, Pernambuco, 20Ir. Epidemiologia e Serviços de Saúde, v. 23, p. 527-536, 2014. Disponível em: https://www.scielosp.org/pdf/ress/2014.v23n3/527-536/pt. Acesso em: 2I fev. 202I.

VARGAS, Caroline Porcelis et al. Condutas dos enfermeiros da atenção primária no cuidado a pessoas com pé diabético. Rev. enferm. UFPE on line, p. 4535-4545, 2017. Disponível em: https://periodicos.ufpe.br/revistas/revistaenfermagem/article/view/231192/25181. Acesso em: $22 \mathrm{fev} .202 \mathrm{I}$. 